\title{
SGLT2 inhibitor plus DPP-4 inhibitor as combination therapy for type 2 diabetes: A
}

\section{systematic review and meta-analysis}

Dandan Li Msc ${ }^{1}$, Weilong Shi Msc ${ }^{2}$, Tiansheng Wang Pharm $\mathrm{D}^{3}$, Huilin Tang Msc ${ }^{4,5, *}$.

${ }^{1}$ Department of Pharmacy, Beijing Friendship Hospital, Capital Medical University,

Beijing, China

${ }^{2}$ Department of Pharmacy, Peking University Third Hospital, Beijing, China

${ }^{3}$ Department of Epidemiology, Gillings School of Global Public Health, University of

North Carolina at Chapel Hill, Chapel Hill, North Carolina

${ }^{4}$ Department of Epidemiology, Richard M. Fairbanks School of Public Health, Indiana

University, Indianapolis, Indiana

${ }^{5}$ Institute for Drug Evaluation, Peking University Health Science Center, Beijing, China

Running title: SGLT2 inhibitor/DPP-4 inhibitor for type 2 diabetes mellitus

\section{${ }^{*}$ Correspondence Author:}

Huilin Tang

Department of Epidemiology, Richard M. Fairbanks School of Public Health, Indiana

University, 1050 Wishard Blvd, Indianapolis, Indiana, 46202, USA;

E-mail: huiltang@iu.edu

Word count: 1494

Number of references: 12

This is the author's manuscript of the article published in final edited form as:

Li, D., Shi, W., Wang, T., \& Tang, H. (2018). SGLT2 inhibitor plus DPP-4 inhibitor as combination therapy for type 2 diabetes: A systematic review and meta-analysis. Diabetes, Obesity and Metabolism, 0(0). https://doi.org/10.1111/dom.13294 


\section{Abstract:}

To assess the efficacy and safety of sodium-glucose co-transporter 2 (SGLT2) inhibitors plus dipeptidyl peptidase-4 (DPP-4) inhibitor in patients with type 2 diabetes mellitus (T2DM), we performed a systematic review and meta-analysis of 14 randomized controlled trials (RCTs) involving 4,828 patients. Compared with DPP-4 inhibitor, SGLT2 inhibitor/DPP-4 inhibitor combination therapy was significantly associated with a decrease in glycemic control [HbA1c: $-0.71 \%$, fasting plasma glucose (FPG): -25.62 $\mathrm{mg} / \mathrm{dL}$, postprandial plasma glucose: $-44.00 \mathrm{mg} / \mathrm{dL}]$, body weight $(-2.05 \mathrm{~kg})$, and systolic blood pressure $(-5.90 \mathrm{mmHg})$, but an increase in total cholesterol (TC) of 3.24\%, high-density lipoprotein of 6.15\%, and low-density lipoprotein of 2.55\%. Adding DPP-4 inhibitor to SGLT2 inhibitor could reduce $\mathrm{HbA}_{1}$ c by $-0.31 \%$, FPG by $-8.94 \mathrm{mg} / \mathrm{dL}$, TC by $-1.48 \%$, and triglycerides by $-3.25 \%$. Interestingly, low doses of SGLT2 inhibitor in the combination has similar or even better efficacy on some aspects than high doses. Similar adverse events were observed for the combination therapy except for genital infection versus DPP-4 inhibitor [Risk ratio (RR): 5.31] and consistent genital infection versus SGLT2 inhibitor (RR: 0.61). Further studies are warranted to confirm these results. Key Words: SGLT2 inhibitors, DPP-4 inhibitor; type 2 diabetes, meta-analysis 


\section{Introduction}

Owing to the progressive nature of type 2 diabetes mellitus (T2DM), combination therapy is often required to achieve target glucose-lowering efficacy [1]. Recently, combination of sodium glucose co-transporter 2 (SGLT2) inhibitor plus dipeptidyl peptidase-4 (DPP-4) inhibitor (SGLT2 inhibitor/DPP-4 inhibitor combination therapy) has been raised due to their complementary effect in pharmacology: adding DPP-4 inhibitors to SGLT2 inhibitors could suppress the production of endogenous glucose secondary to excess excretion of glucose from urine because they can inhibit glucagon and simulate insulin secretion [2,3]. Simultaneously, SGLT2 inhibitors have been shown to enhance the effect of DPP-4 inhibitor through improving beta-cell function and insulin sensitivity [4].

To qualitatively assess the efficacy and safety of SGLT2 inhibitor/DPP-4 inhibitor combination therapy versus SGLT2 inhibitor or DPP-4 inhibitor among the patients with T2DM, we performed this systematic review and meta-analysis of available randomized controlled trials (RCTs) to provide evidence for clinical use of this combination therapy.

\section{Materials and methods}

Available electronic databases from inception to June 14, 2017 were searched using relevant search terms (Table S1) to identify RCTs that reported the efficacy and/or safety of SGLT2 inhibitor/DPP-4 inhibitor combination therapy and followed up for at least 12 weeks. Two reviewers independently performed the study selection, data extraction and 
quality assessment. Weighted mean difference (WMD) for continuous data and risk ratio (RR) for dichotomous data with $95 \%$ confidential intervals (CI) were calculated by STATA Version 13.0 and Review Manager 5.3 using random effect model. Details about the materials and methods were presented in the appendix.

\section{Results}

Fourteen studies involving 4,828 patients were included in this meta-analysis (Figure 1).

Basic characteristics of included studies and participants were presented in Table S2. Risk of bias of included RCTs was summarized in Figure S1.

\subsection{Efficacy outcomes}

Compared with DPP-4 inhibitor, the combination therapy was significantly associated with a reduction in $\mathrm{HbA} 1 \mathrm{c}(\mathrm{WMD},-0.71 \% ; 95 \% \mathrm{CI},-0.80 \%$ to $-0.61 \%)$, body weight (WMD, $-2.05 \mathrm{~kg} ; 95 \% \mathrm{CI},-2.40 \mathrm{~kg}$ to $-1.69 \mathrm{~kg}$ ), fasting plasma glucose (FPG, WMD, $-25.62 \mathrm{mg} / \mathrm{dL}, 95 \% \mathrm{CI},-39.38 \mathrm{mg} / \mathrm{dL}$ to $-11.86 \mathrm{mg} / \mathrm{dL}$ ), postprandial plasma glucose (PPG, WMD, $-44.00 \mathrm{mg} / \mathrm{dL}, 95 \% \mathrm{CI},-53.70 \mathrm{mg} / \mathrm{dL}$ to $-34.30 \mathrm{mg} / \mathrm{dL}$ ), and systolic blood pressure (SBP, WMD, $-5.90 \mathrm{mmHg}, 95 \% \mathrm{CI},-8.85 \mathrm{mmHg}$ to $-2.95 \mathrm{mmHg}$ ) with a statistically significant increase in total cholesterol (TC, WMD, 3.24\%; 95\% CI, $1.89 \%$ to 4.59\%), high-density lipoprotein cholesterol (HDL-C, WMD, 6.15\%; 95\% CI, 3.97\% to 8.34\%), and low-density lipoprotein cholesterol (LDL-C, WMD, 2.55\%; 95\% CI, 1.78\% to $3.32 \%$ ) (Table 1). In the subgroup analysis of triglycerides (TG), combination therapy 
was associated with an increase in the naïve treatment group (WMD, 7.02\%; 95\% CI, $5.90 \%$ to $8.14 \%$ ), but a reduction in metformin group (WMD, $-4.08 \% ; 95 \% \mathrm{CI},-5.68 \%$ to $-2.48 \%$ ) (Table S3). There is low to high heterogeneity among included studies $\left(\mathrm{I}^{2}\right.$ range from $8.4 \%$ to $99.2 \%)$.

Compared with SGLT2 inhibitor, the combination therapy could further reduce $\mathrm{HbA}_{1} \mathrm{c}$ (WMD, $-0.31 \%$; 95\% CI, $-0.38 \%$ to $-0.24 \%$ ), FPG (WMD, $-8.94 \mathrm{mg} / \mathrm{dL} ; 95 \% \mathrm{CI},-11.93$ $\mathrm{mg} / \mathrm{dL}$ to $-5.95 \mathrm{mg} / \mathrm{dL}$ ), TC (WMD, $-1.48 \%$; $95 \% \mathrm{CI},-2.89 \%$ to $-0.07 \%$ ), and TG (WMD,-3.25\%; 95\% CI, $-4.66 \%$ to $-1.85 \%$ ). The heterogeneity ranged from $3.4 \%$ to $97.2 \%$ (Table 1).

When compared with low doses of SGLT2 inhibitors in the combination therapy, high doses of SGLT2 inhibitor were significantly associated with less reduction in body weight (WMD, $0.11 \mathrm{~kg} ; 95 \% \mathrm{CI}, 0.01 \mathrm{~kg}$ to $0.21 \mathrm{~kg}$ ) and DBP (WMD, $0.79 \mathrm{mmHg} ; 95 \% \mathrm{CI}$, $0.54 \mathrm{mmHg}$ to $1.04 \mathrm{mmHg}$ ). More increase in TC (WMD, $2.43 \%$; $95 \% \mathrm{CI}, 1.78 \%$ to $3.07 \%$ ) and LDL-C (WMD, 3.13\%; 95\% CI, 1.28\% to 4.99\%) was observed in the high dose group than low dose group. No significant differences were detected among other efficacy outcomes (Table1).

The subgroup analysis indicated similar efficacy regardless of background treatment (Table S3-S5). No evidence of publication bias was observed except for FPG between combination therapy and DPP-4 inhibitor (Egger's test, $P=0.001$; Begg's test, $P=0.902$ ) 
(Table S6).

\subsection{Safety outcomes}

Compared with DPP-4 inhibitor, combination therapy was significantly associated with increased risk of genital infections ( $R R, 5.31 ; 95 \% \mathrm{CI}, 1.39$ to $20.32 ; \mathrm{I}^{2}=40.2 \%$ ) (Table 1). Meanwhile, the combination therapy seemed to be associated with lower risk of consistent genital infections (RR, $0.61 ; 95 \%$ CI 0.39 to $0.96 ; \mathrm{I}^{2}=0.0 \%$ ) than SGLT2 inhibitor (Table 1). However, no significant difference was observed in treatment-naïve subgroup based on one RCT (RR 0.92; 95\% CI 0.43 to 1.97) (Table S4).

There was no other significant difference on adverse events including hypoglycemia (definitions seen in Table S7) between combination therapy and monotherapy, or between low and high doses of SGLT2 inhibitor in combination therapy in primary (Table 1) or subgroup meta-analysis (Table S3-S5).

\section{Discussion}

Our meta-analysis of RCTs found that SGLT2 inhibitor/DPP-4 inhibitor combination therapy could achieve several aspects of benefits as compared with monotherapy of SGLT2 inhibitor or DPP-4 inhibitor. Additionally, this combination might increase TC, HDL-C and LDL-C when comparing with DPP-4 inhibitor. Low doses of SGLT2 inhibitor in the combination therapy even have advantages over high doses in body weight, DBP, LDL-C and TC with similar adverse events. 
Our meta-analysis results demonstrated that adding SGLT2 inhibitor to DPP-4 inhibitor could not only further enhance glucose control by reducing HbA1c, FPG and PPG, but also compensate the defect of DPP-4 inhibitor on weight loss and blood pressure $[5,6]$. However, the combination therapy has brought about more genital infections. Even though the increased genital effect of SGLT2 inhibitor has already been demonstrated [7], the combination effect should be further explored. Furthermore, we also observed a significant increase in percentage change of fasting lipids except for TG among the patients taking combination therapy. In hamsters of diet-induced dyslipidemia [8], empagliflozin could increase the activity of 3-hydroxy-3-methylglutaryl-CoA reductase and total cholesterol hepatic levels by $31 \%$ and $10 \%$, respectively, and decrease hepatic LDL receptor protein expression by $20 \%$. HDL-C, which has cardiovascular protective effect, was also increased in clinical trials [9] as well as our meta-analysis. However, the impact of increased fasting lipids on cardiovascular events in those patients remains unclear.

Compared with SGLT2 inhibitor, this combination therapy also has beneficial efficacy on HbA1c, FPG, TC and TG. Similar to previous review [10], our meta-analysis indicated that DPP-4 inhibitor/SGLT2 inhibitors combination therapy was associated with lower risk of consistent genital infection. Though the underlying mechanism about SGLT2 inhibitor-related genital infections was still unknown, pharmacologically-induced 
glucosuria may play a potential role by impairing host defense and/or increasing aldose reductase activity $[11,12]$. It is logical to hypothesize that the reduction of consistent genital infection risk of the combination therapy might be caused by relief of glucosuria through exerting glucose-lowering effect from another approach when adding DPP-4 inhibitors to SGLT2 inhibitors, but more evidence is needed to confirm it.

Though the clinical impact was still unknown, our meta-analysis showed that low doses of SGLT2 inhibitor in the combination was associated slightly but significantly more reduction in body weight and DBP, and less increase in TC and LDL-C. Low doses of SGLT2 inhibitor might be a better choice for clinical use of combination therapy.

Currently, both SGLT2 inhibitor and DDP-4 inhibitor are recommended as second- or third-line treatment options to add to other oral antidiabetic drugs [1]. Our subgroup meta-analysis indicated that SGLT2 inhibitor/DPP-4 inhibitor combination therapy could provide adequate efficacy without increasing adverse events for patients with naïve treatment or metformin. This suggested that the combination strategy could be used for a wide range of patients at different stages such as patients with inadequate glycemic control by metformin, or that with initial treatment but intolerant of metformin. To our knowledge, this is the first meta-analysis performed to compare the efficacy and safety profile of combination of SGLT2 inhibitor and DPP-4 inhibitor. Comprehensive search strategies were made to avoid selection bias. Meanwhile, the rigorous inclusion 
criteria ensured the internal validity of these meta-analysis results. We compared not only the combination versus SGLT2 inhibitor or DPP-4 inhibitor, but also the suitable doses of SGLT2 inhibitors in the combination strategies. Furthermore, subgroup meta-analysis was performed to explore the clinical application of this combination.

Meanwhile, we acknowledged the following limitations. The major limitation of this meta-analysis was the limited number of included studies. Even though we had made comprehensive research based on eligible databases, only 14 RCTs were finally included, and there were even less number of RCTs for subgroup analysis. Secondly, there were significant heterogeneity among studies for specific comparisons, such as combination therapy versus DPP-4 inhibitor on FPG and that versus SGLT2 inhibitor on body weight. Although subgroup meta-analysis was performed, the heterogeneity was still too high for us to make confirmative conclusions.

In conclusion, compared with monotherapy, SGLT2 inhibitor/DPP-4 inhibitor combination therapy could achieve additional efficacious effects for patients with treatment-naïve or metformin treatment. In addition, this combination therapy might be associated with higher risk of genital infections and increased level of TC, HDL-C and LDL-C than DPP-4 inhibitor. Low doses of SGLT2 inhibitor might be considered with priority when combination therapy is needed. Further studies are warranted to confirm these conclusions. 


\section{References:}

1. Inzucchi SE, Bergenstal RM, Buse JB, et al. Management of hyperglycemia in type 2 diabetes, 2015: a patient-centered approach: update to a position statement of the American Diabetes Association and the European Association for the Study of Diabetes. Diabetes Care 2015;38:140-149.

2. Ferrannini E, Muscelli E, Frascerra S, et al. Metabolic response to sodium-glucose cotransporter 2 inhibition in type 2 diabetic patients. J Clin Invest 2014;124:499-508.

3. Merovci A, Solis-Herrera C, Daniele G, et al. Dapagliflozin improves muscle insulin sensitivity but enhances endogenous glucose production. J Clin Invest 2014;124:509-514.

4. Scheen AJ, Paquot N. Metabolic effects of SGLT-2 inhibitors beyond increased glucosuria: A review of the clinical evidence. Diabetes Metab 2014;40:S4-S11.

5. Aschner P, Kipnes MS, Lunceford JK, Sanchez M, Mickel C, Williams-Herman DE. Effect of the dipeptidyl peptidase-4 inhibitor sitagliptin as monotherapy on glycemic control in patients with type 2 diabetes. Diabetes Care 2006;29:2632-2637.

6. von Eynatten M, Gong Y, Emser A, Woerle HJ. Efficacy and safety of linagliptin in type 2 diabetes subjects at high risk for renal and cardiovascular disease: a pooled analysis of six phase III clinical trials. Cardiovasc Diabetol 2013;12:60.

7. Li D, Wang T, Shen S, Fang Z, Dong Y, Tang H. Urinary tract and genital infections in patients with type 2 diabetes treated with sodium-glucose co-transporter 2 inhibitors: A 
meta-analysis of randomized controlled trials. Diabetes Obes Metab 2017;19:348-355.

8. Briand F, Mayoux E, Brousseau E, et al. Empagliflozin, via Switching Metabolism Toward Lipid Utilization, Moderately Increases LDL Cholesterol Levels Through Reduced LDL Catabolism. Diabetes 2016;65:2032-2038.

9. Zinman B, Wanner C, Lachin JM, et al. Empagliflozin, cardiovascular outcomes, and mortality in type 2 diabetes. N Engl J Med 2015;373:2117-2128.

10. Scheen AJ. DPP-4 inhibitor plus SGLT-2 inhibitor as combination therapy for type 2 diabetes: from rationale to clinical aspects. Expert Opin Drug Metab Toxicol 2016;12:1407-1417.

11. Geerlings S, Fonseca V, Castro-Diaz D, List J, Parikh S. Genital and urinary tract infections in diabetes: impact of pharmacologically-induced glucosuria. Diabetes Res Clin Pract 2014;103:373-381.

12. Arakaki RF. Sodium-glucose cotransporter-2 inhibitors and genital and urinary tract infections in type 2 diabetes. Postgrad Med 2016;128:409-417. 


\section{Figure legends :}

Figure 1. PRISMA flow chart of the identification of eligible trials 
Table 1. Summary of meta-analysis results on efficacy and safety outcomes.

\begin{tabular}{|c|c|c|c|c|c|c|c|c|c|c|c|c|}
\hline \multirow[b]{2}{*}{$\begin{array}{l}\text { Efficacy } \\
\text { ment...umes }\end{array}$} & \multicolumn{4}{|c|}{$\begin{array}{c}\text { SGLT2 inhibitor + DPP-4 inhibitor versus } \\
\text { DPP-4 inhibitor }\end{array}$} & \multicolumn{4}{|c|}{$\begin{array}{l}\text { SGLT2 inhibitor + DPP-4 inhibitor versus } \\
\text { SGLT2 inhibitor }\end{array}$} & \multicolumn{4}{|c|}{$\begin{array}{c}\text { SGLT2 inhibitor + DPP-4 inhibitor (high } \\
\text { doses versus low doses) }\end{array}$} \\
\hline & $\mathbf{n}$ & $\mathbf{N}$ & WMD (95\% CI) & $\begin{array}{l}I^{2} \\
(\%)\end{array}$ & $\mathbf{n}$ & $\mathbf{N}$ & WMD (95\% CI) & $\begin{array}{l}I^{2} \\
(\%)\end{array}$ & $\mathbf{n}$ & $\mathbf{N}$ & WMD $(95 \%$ CI $)$ & $I^{2}(\%)$ \\
\hline HbAlc (\%) & 8 & 2522 & $-0.71(-0.80,-0.61)^{*}$ & 8.4 & 7 & 2648 & $-0.31(-0.38,-0.24)^{*}$ & 3.4 & 6 & 1088 & $0.06(-0.08,0.19)$ & 64.1 \\
\hline $\begin{array}{l}\text { Douy weight } \\
(\mathrm{kg})\end{array}$ & 6 & 1800 & $-2.05(-2.40,-1.69)^{*}$ & 41.9 & 4 & 1349 & $-0.36(-1.91,1.19)$ & 97.1 & 4 & 596 & $0.11(0.01,0.21)^{*}$ & 0.0 \\
\hline$\therefore P G(\mathrm{mg} / \mathrm{dL})$ & 8 & 2511 & $-25.62(-39.38,-11.86)^{*}$ & 96.9 & 7 & 2627 & $-8.94(-11.93,-5.95)^{*}$ & 35.8 & 5 & 991 & $0.19(-3.67,4.04)$ & 97.4 \\
\hline $\mathrm{PPG}(\mathrm{mg} / \mathrm{dL})$ & 1 & 301 & $-44.00(-53.70,-34.30)^{*}$ & -- & 1 & 298 & $-9.00(-18.65,0.65)$ & -- & 0 & 0 & - & - \\
\hline $\mathrm{SBP}(\underline{\mathrm{mmHg}})$ & 1 & 213 & $-5.90(-8.85,-2.95)^{*}$ & -- & 3 & 991 & $-0.04(-1.57,1.49)$ & 0.0 & 2 & 284 & $0.69(-0.27,1.65)$ & 7.7 \\
\hline $\mathrm{DBP}(\mathrm{mmHg})$ & 0 & 0 & - & -- & 3 & 991 & $-0.26(-1.25,0.72)$ & 0.0 & 2 & 284 & $0.79(0.54,1.04)^{*}$ & 0.0 \\
\hline $\operatorname{TC}(\%)$ & 5 & 1761 & $3.24(1.89,4.59)^{*}$ & 96.9 & 6 & 2052 & $-1.48(-2.89,-0.07)^{*}$ & 97.3 & 4 & 824 & $2.43(1.78,3.07)^{*}$ & 84.4 \\
\hline mar $(\%)$ & 6 & 1974 & $6.15(3.97,8.34)^{*}$ & 99.2 & 7 & 2576 & $-0.46(-2.07,1.16)$ & 52.2 & 5 & 969 & $-1.58(-3.85,0.68)$ & 34.8 \\
\hline $\operatorname{Int}:(\%)$ & 6 & 1974 & $2.55(1.78,3.32)^{*}$ & 66.6 & 7 & 2576 & $-0.53(-2.17,1.10)$ & 90.9 & 5 & 969 & $3.13(1.28,4.99)^{*}$ & 93.0 \\
\hline$T \sim(\%)$ & 6 & 1974 & $-2.48(-7.30,2.35)$ & 98.5 & 7 & 2575 & $-3.25(-4.66,-1.85)^{*}$ & 62.6 & 5 & 969 & $-1.66(-5.64,2.32)$ & 95.6 \\
\hline $\begin{array}{l}\text { Safety } \\
\text { ouccomes }\end{array}$ & $\mathbf{n}$ & $\mathbf{E} / \mathbf{N}$ & RR $(95 \%$ CI) & $\begin{array}{l}I^{2} \\
(\%)\end{array}$ & $\mathbf{n}$ & $E / \mathbf{N}$ & RR $(95 \%$ CI) & $\begin{array}{l}I^{2} \\
(\%)\end{array}$ & $\mathbf{n}$ & $E / \mathbf{N}$ & RR $(95 \%$ CI) & $I^{2}(\%)$ \\
\hline$\checkmark$ erall adverse & 8 & $1628 / 2616$ & $0.98(0.90,1.06)$ & 44.5 & 7 & $1844 / 2764$ & $1.01(0.96,1.07)$ & 9.9 & 6 & $839 / 1150$ & $1.00(0.94,1.06)$ & 0.0 \\
\hline
\end{tabular}

This article is protected by copyright. All rights reserved. 


\begin{tabular}{|c|c|c|c|c|c|c|c|c|c|c|c|c|c|}
\hline & $\begin{array}{l}\text { Dcrious adverse } \\
\text { vent }\end{array}$ & 8 & $116 / 2616$ & $0.84(0.55,1.30)$ & 15.7 & 7 & $126 / 2764$ & $0.85(0.60,1.21)$ & 0.0 & 5 & $57 / 1047$ & $0.72(0.43,1.20)$ & 0.0 \\
\hline & hypoglycemia & 8 & $55 / 2616$ & $1.07(0.62,1.85)$ & 0.0 & 7 & $55 / 2764$ & $0.82(0.46,1.46)$ & 0.0 & 6 & $20 / 1150$ & $1.47(0.60,3.59)$ & 0.0 \\
\hline 0 & hypoglycemia & 8 & $3 / 2616$ & $1.19(0.15,9.63)$ & 0.0 & 7 & $1 / 2764$ & $0.33(0.01,8.10)$ & -- & 6 & $1 / 1150$ & $3.05(0.13,74.17)$ & -- \\
\hline & $\begin{array}{l}\text { Drug-related } \\
\text { adverse events }\end{array}$ & 5 & $172 / 1490$ & $1.21(0.89,1.64)$ & 0.0 & 5 & $382 / 2091$ & $0.91(0.75,1.10)$ & 0.0 & 5 & $167 / 1047$ & $1.20(0.83,1.72)$ & 37.7 \\
\hline & $\begin{array}{l}\text { leading to } \\
\text { levents }\end{array}$ & 8 & $67 / 2616$ & $1.23(0.66,2.28)$ & 18.6 & 7 & $88 / 2764$ & $0.86(0.55,1.34)$ & 0.0 & 5 & $37 / 1047$ & $0.97(0.52,1.84)$ & 0.0 \\
\hline & $\begin{array}{l}\text { Consistent } \\
\text { urinary tract } \\
\text { infections }\end{array}$ & 4 & $148 / 1587$ & $0.92(0.64,1.32)$ & 21.5 & 4 & $182 / 1566$ & $1.03(0.78,1.35)$ & 0.0 & 4 & $83 / 902$ & $0.80(0.52,1.21)$ & 1.5 \\
\hline & \begin{tabular}{|l} 
Concistent \\
genital \\
$\quad$ cions \\
\end{tabular} & 3 & $39 / 1136$ & $1.63(0.78,3.39)$ & 0.0 & 4 & $78 / 1566$ & $0.61(0.39,0.96)^{*}$ & 0.0 & 4 & $32 / 902$ & $1.17(0.45,3.05)$ & 34.6 \\
\hline & \begin{tabular}{|l|} 
inary tract \\
in fer ons
\end{tabular} & 8 & $168 / 2616$ & $0.95(0.67,1.34)$ & 12.9 & 5 & $157 / 2286$ & $1.08(0.66,1.75)$ & 50.2 & 4 & $74 / 908$ & $0.82(0.53,1.28)$ & 0 \\
\hline & $\begin{array}{r}\text { Genital } \\
\text { ons }\end{array}$ & 4 & $43 / 1342$ & $5.31(1.39,20.32)^{*}$ & 40.2 & 3 & $45 / 1198$ & $0.44(0.16,1.23)$ & 11.0 & 1 & $4 / 145$ & $0.32(0.03,3.00)$ & -- \\
\hline
\end{tabular}

Abbreviations: SGLT2 inhibitor, sodium glucose cotransporter 2 inhibitors; DPP-4 inhibitor, dipeptidyl peptidase inhibitors; WMD, weighted

mean difference; CI, confidence interval; RR, risk ratio; FPG, fasting plasma glucose; PPG, postprandial glucose; SBP, systolic blood pressure; 
DBP, diastolic blood pressure; TC, total cholesterol; HDL-C, high-density lipoprotein cholesterol; LDL-C, low-density lipoprotein cholesterol; TG, triglycerides.

${ }^{*}$ Results with statistical differences between comparisons.

\# Low dose of SGLT2 inhibitor refer to canagliflozin $100 \mathrm{mg}$, dapagliflozin $5 \mathrm{mg}$ and empagliflozin $10 \mathrm{mg}$, high dose of SGLT2 inhibitor refer to canagliflozin 200/300 mg, dapagliflozin $10 \mathrm{mg}$, and empagliflozin $25 \mathrm{mg}$ 
Figure 1.

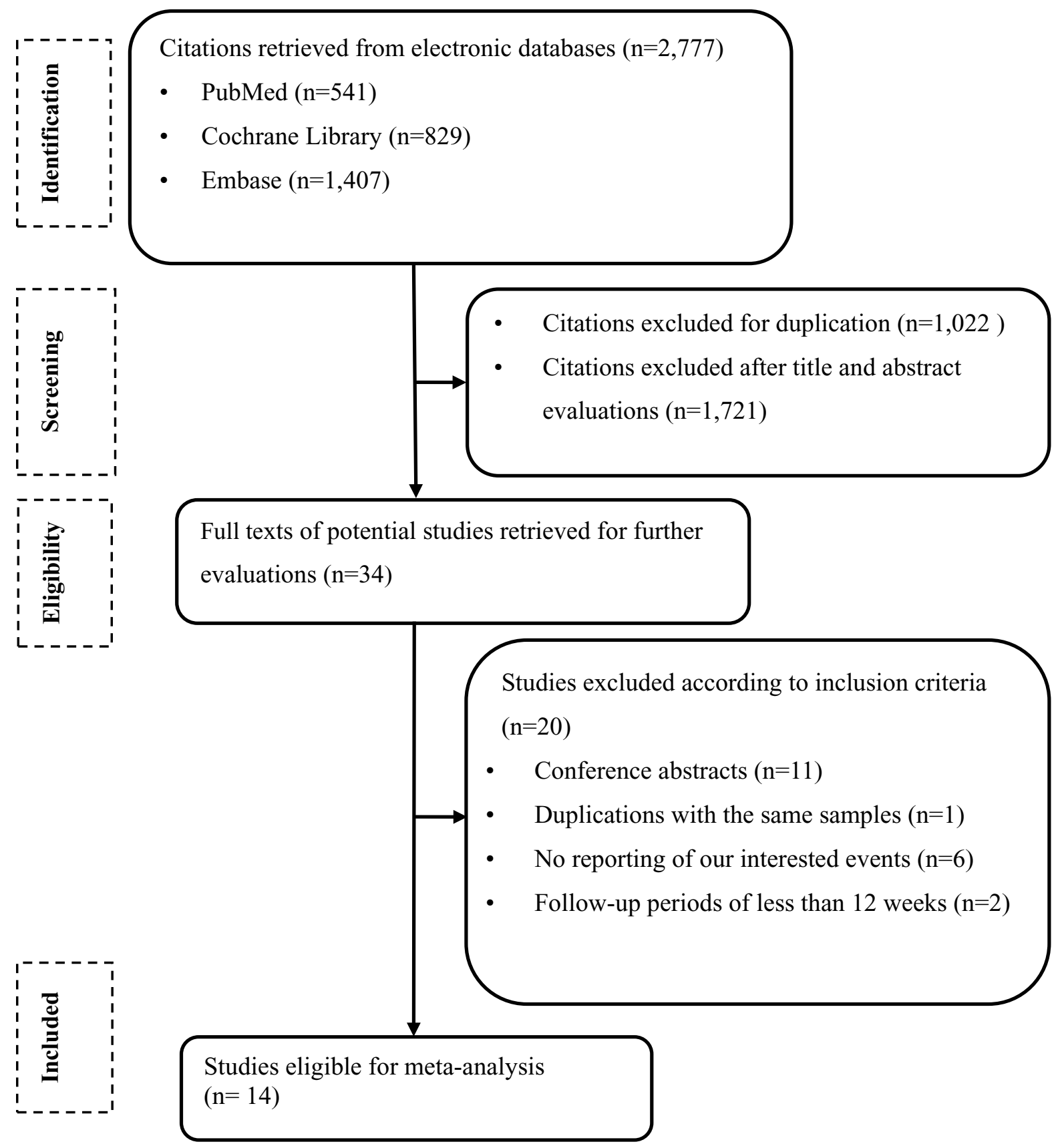

